\title{
A Study on the End-Effector Exchange Mechanism of a Space Robot
}

\author{
Rui $\mathrm{Qu}^{1}$, Yuto Takei ${ }^{1}$, Mitsushige Oda ${ }^{1}$, Hiroki Nakanishi ${ }^{1}$, Armin Wedler ${ }^{2}$ and Kent Yoshikawa $^{1}$ \\ 1. Department of Mechanical and Aerospace Engineering, Tokyo Institute of Technology, Tokyo 1528550, Japan \\ 2. RMC (Robotics and Mechatronics Center), DLR (German Aerospace Center), Oberpfaffenhofen 82234, Germany
}

\begin{abstract}
This study investigates the necessity for space robots to independently exchange their end-effectors due to the contradiction that exists between the requirements of various robots in space missions and the payload capacity limits of rockets. The results of this study summarize the system requirements for a new end-effector exchange mechanism, including compact size, misalignment tolerance, and regolith tolerance. This is followed by the development of a prototype model with a set of test apparatus. Then the function of the prototype is verified, the prototype is optimized, and the relation between docking force and misalignment is examined through operation tests.
\end{abstract}

Key words: Space robot, end-effector exchange mechanism, operation test.

\section{Introduction}

Future space missions involve the construction and assembly of large space structures, including a lunar base and an SSPS (space solar power system), and it is impossible for human beings to operate these missions without the support of robots. The missions require various robots, whose spare components are necessary because manual operation and maintenance of robots in space is also unavailable. However, a rocket continues to be the only method of transportation to space. The limit of the payload capacity of a rocket necessitates the minimization of equipment to the maximum possible extent.

This restriction leads to a concept in which a robot is divided into a robot arm and an end-effector. It is not necessary to ensure a robot arm for every end-effector. However, an EEEM (end-effector exchange mechanism) is necessary to connect the robot arm and the end-effector from the mechanical side and electrical side, as an interface. Hence, the Tokyo Institute of Technology and German Space Center

Corresponding author: Rui Qu, master, doctoral student, research field: space robotics.
(DLR) commenced a collaborated project on EEEM.

Several EEEMs have been developed in extant studies. The following section describes a detailed survey of two EEEMs previously developed for space robots, which correspond to the focus of the present study.

ETS-VII (Engineering Test Satellite VII) demonstrated robot arm operation in several missions, from 1997 to 1999. Three types of grapple fixtures (Fig. 1 (a)) exist between the end-effector of the robot arm (Fig. 1 (b)) and the payloads, to play the role of an EEEM, to ensure that the robot arm can hold different types of payloads.

Three ball-shaped alignment cones exist on the grapple fixture, for misalignment canceling. Additionally, three sets of finger covers aid fingers on the tip of the end-effector in accurately grasping the grapple fixture, and three latching mechanisms aid in holding the grapple fixture [1, 2].

An SPDM (special purpose dexterous manipulator), also referred to as "Dextre", was launched in February 2008. It is the last component of Canada's MSS (mobile servicing system) for the ISS (international space station). 


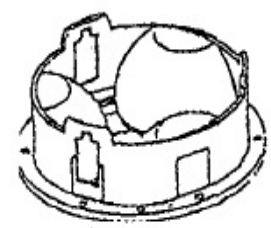

(Standard GPF)

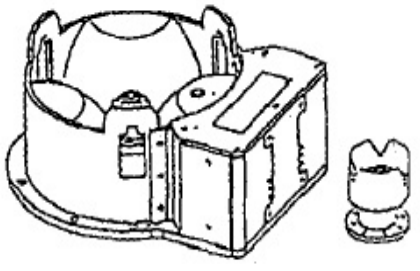

(GPF with connector) (Small GPF)

(a) Grapple fixtures

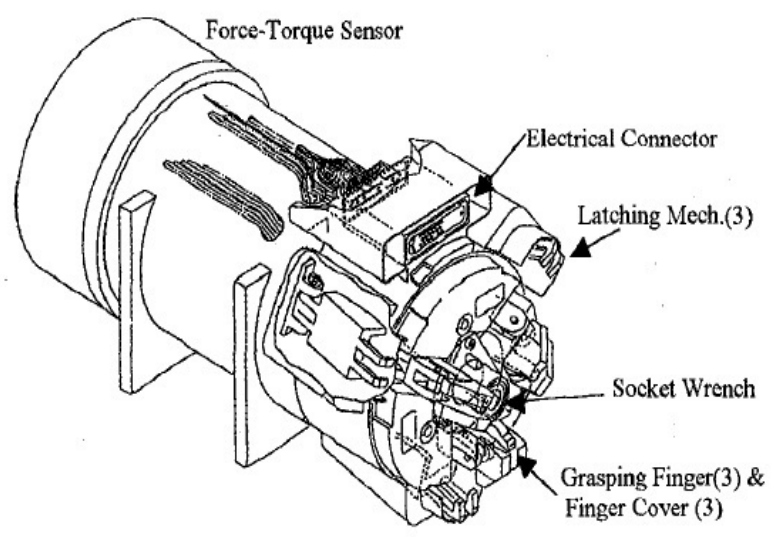

(b) End-effector

Fig. 1 ETS-VII grapple fixtures and an end-effector. (C) JAXA.

Dextre's arm connects to the end-effectors through an OTCM (ORU-tool change out mechanism). For example, the OTCM possesses an internal gripper mechanism to drive its two jaws which can capture a standard micro interface on the bottom of the SARAH (self-adapting robotic auxiliary hand). Subsequently, a socket head of the OTCM extends, to engage a captive hexagonal bolt head. Therefore, the OTCM can control the fingers of SARAH through the socket head and a switching mechanism. Additionally, a pair of lights and a bore sight camera enable operators to operate with a lower degree of misalignment [3, 4]. Fig. 2 shows mechanism of OTCM and interaction of SPDM/OTCM and SARAH.

Based on the aforementioned background of this study, the study objectives are summarized as follows:

- To investigate the system requirements for a new EEEM that is superior to extant EEEMs;

- To develop a new EEEM, including a prototype model and a set of test apparatus for it;

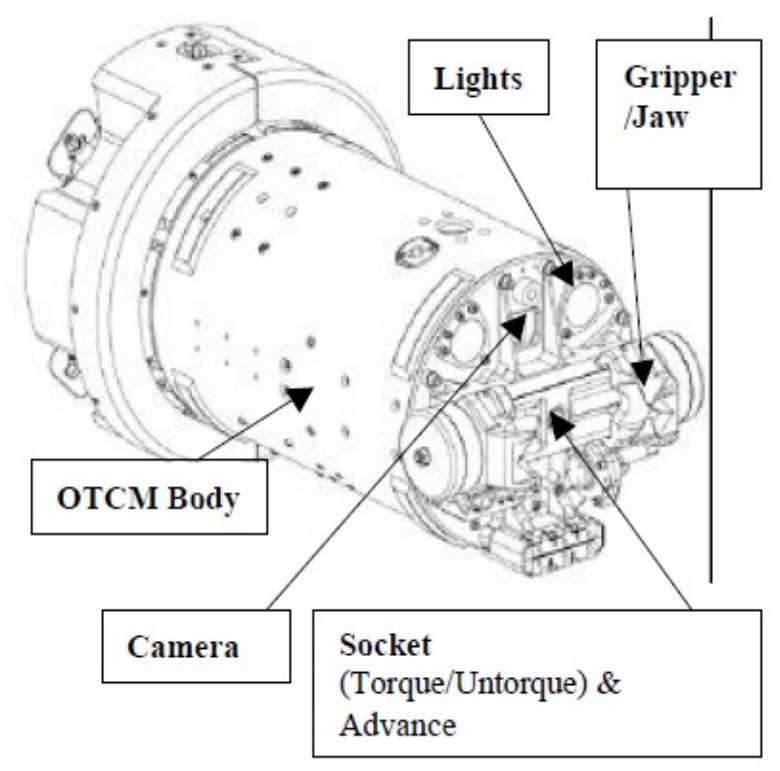

(a) OTCM

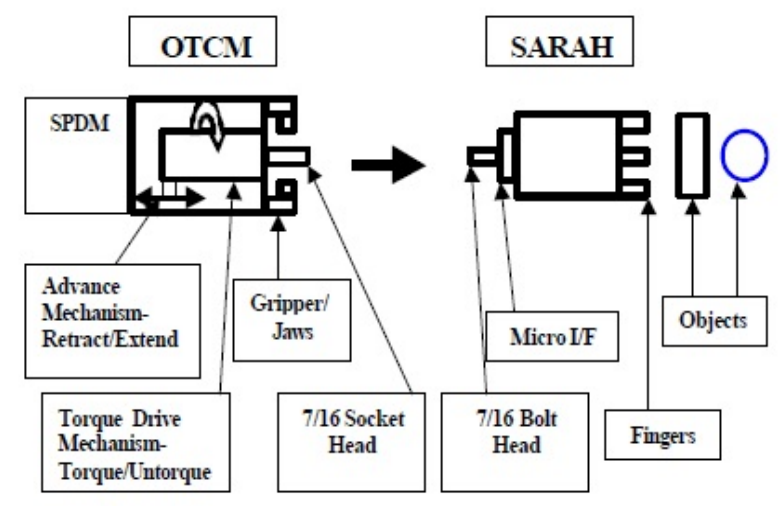

(b) Interaction of SPDM/OTCM and SARAH

Fig. 2 OTCM and the interaction of SPDM/OTCM and SARAH. CCSA.

- To operate a function verification test and an optimization test, and to study a method of misalignment canceling.

\section{System Requirements}

Requirements for a new EEEM that is superior to extant EEEMs are summarized in this section.

(1) For the purpose of cost saving, a prototype model is initially developed with a diameter that is less than $100 \mathrm{~mm}$. It is intended for using as an assembling robot for a construction mission.

(2) A requirement for misalignment tolerance is that both position misalignment and rotation misalignment 
should exist without an external force and support of camera feedback during capture.

In order to satisfy the above requirements, we propose a concept of EEEM by using a spring force. A spring-capture mechanism is applied to ensure accurate docking and to increase misalignment tolerance.

Fig. 3 shows an open mode and a closed mode of the spring mechanism. The left side illustrates the open mode while the spring is in a natural state. The right side illustrates the closed mode while the spring is restricted by an external mechanism.

Fig. 4 shows the concept of the new EEEM. The left side of the figure shows that the spring mechanism is in the open mode and can capture the end-effector with a high misalignment tolerance. The right side of the figure shows that the spring mechanism is in the closed mode and can lock the end-effector on the robot arm.

(3) The electrical interface is set at the center of whole mechanism as a minimal solution for the regolith problem.

Otherwise, the installation of several electromagnetic mechanisms on the docking surface is proposed, to remove the regolith with the support of physical method. However, this concept is not verified in this study due to limited test conditions and this will be explored in a future study.

Table 1 shows a comparison of the proposed new EEEM with the extant EEEM.

\section{Development of the Test Apparatus}

A prototype model is developed after discussing the concepts to satisfy the system requirements.
A prototype model of the active side is shown in Fig. 5. There are two linear motion guides that are driven by the motor on the active side of the EEEM. One is used to guide the holding plate for springs and the other is used to guide holding plates for fixing-pins. It should be noted that fixing-pins play the role of an external mechanism to restrict the springs. First, the springs come out and open to capture the passive side, the fixing-pins then come out to close the springs, and they are eventually simultaneously retracted to lock the passive side onto the active side. Fig. 6 shows the capture process.

The main parameters of the prototype model (active side) are given in Table 2.

The concept of the test apparatus is shown in Fig. 7.
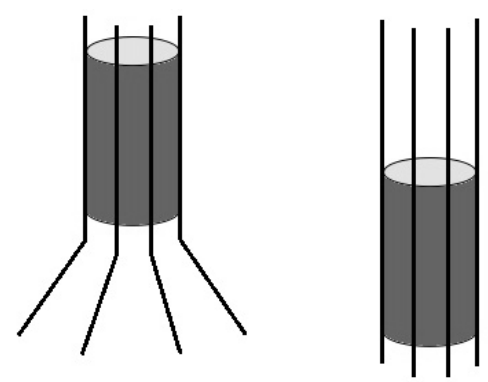

Fig. 3 Two modes of the spring mechanism.

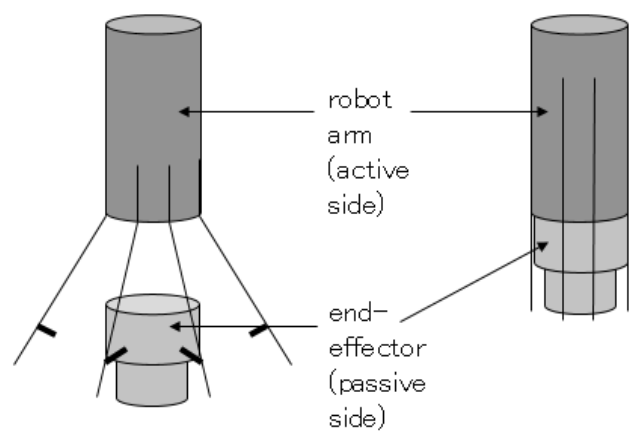

Fig. 4 Concept of the new EEEM.

Table 1 Comparison of the three EEEMs.

\begin{tabular}{|l|l|l|l|}
\hline & New EEEM & ETS-VII & Dextre \\
\hline Operation environment & $\begin{array}{l}\text { On-orbit service \& planet } \\
\text { exploration }\end{array}$ & On-orbit service & On-orbit service \\
\hline Size (diameter) & Less than $100 \mathrm{~mm}$ & Approximately $130 \mathrm{~mm}$ & Approximately $180 \mathrm{~mm}$ \\
\hline Type of locking & Latching mechanism & Latching mechanism & $\begin{array}{l}\text { Bolt/socket \& latching } \\
\text { mechanism }\end{array}$ \\
\hline $\begin{array}{l}\text { Method of misalignment } \\
\text { canceling }\end{array}$ & $\begin{array}{l}\text { Increasing capture } \\
\text { range by using a spring }\end{array}$ & Ball-shaped alignment cone & $\begin{array}{l}\text { Gripper/jaws and camera } \\
\text { feedback }\end{array}$ \\
\hline Position of electrical interface & Center & Outside & Outside \\
\hline
\end{tabular}


Table 2 Main parameters of the prototype model (active side).

\begin{tabular}{|l|l|}
\hline Size (springs and pins are retracted) & $131 \mathrm{~mm} \times 140 \mathrm{~mm} \times 405 \mathrm{~mm}$ \\
\hline Maximum length (springs and pins are deployed) & $475 \mathrm{~mm}$ \\
\hline External diameter of the capture portion & $90 \mathrm{~mm}$ \\
\hline Internal diameter of the capture portion & $38 \mathrm{~mm}$ \\
\hline Weight & $3,437 \mathrm{~g}$ \\
\hline Motor type & Maedler 47520113 \\
\hline Motor power & $36 \mathrm{~W}$ \\
\hline Maximum distance between two guide plates & $74 \mathrm{~mm}$ \\
\hline
\end{tabular}

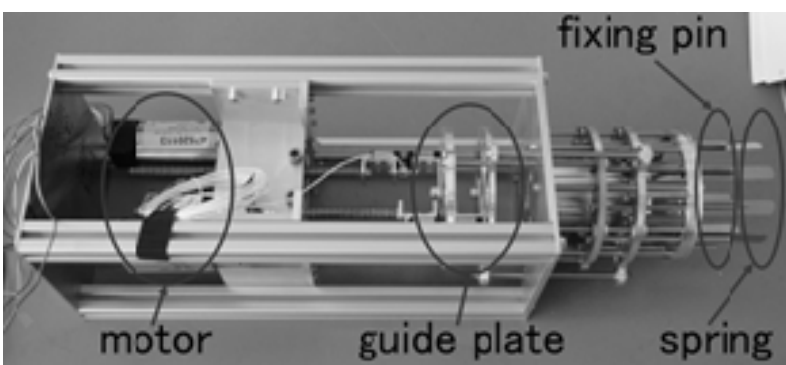

Fig. 5 Mechanism of the prototype model (active side).
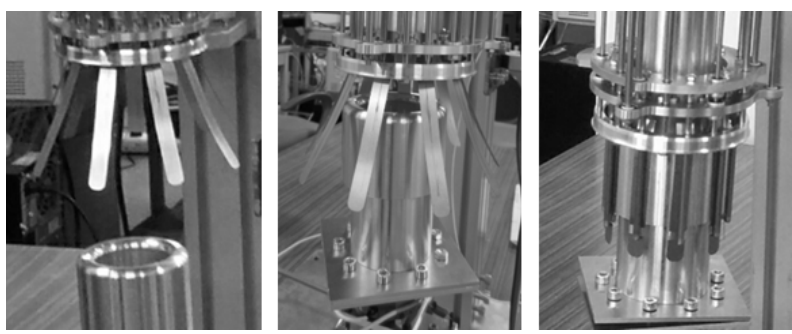

Fig. 6 Capture process of the fingers.

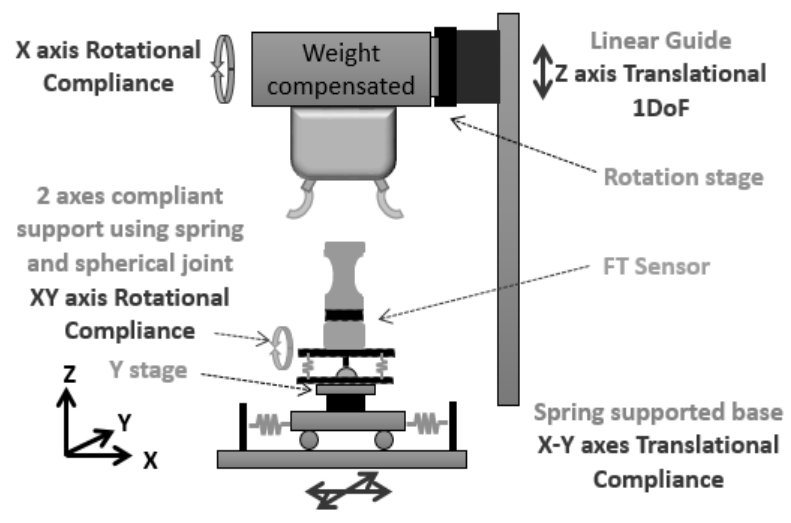

Fig. 7 Concept of the test apparatus.

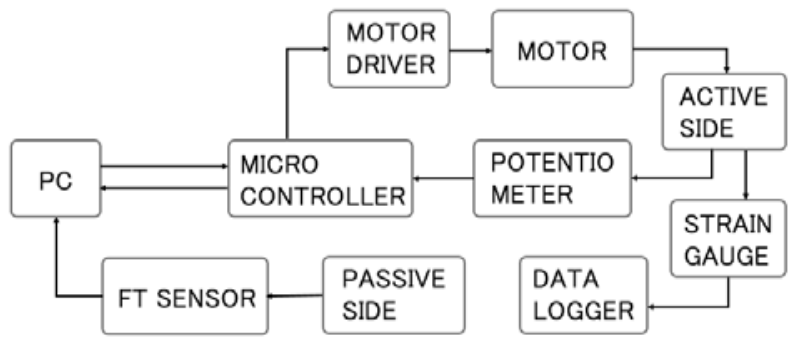

Fig. 8 A block diagram of the electrical control circuit.
A compliant base connected to the passive side is fabricated by using spring and spherical joints to provide the capability to rotate several degrees in the direction of two axes. Additionally, a roll stage connected to the active side, which introduces rotation misalignment, while a Y stage connected to the passive side introduces position misalignment.

An electrical control circuit is developed, as shown in Fig. 8.

An H-bridge motor driver and an Arduino micro-controller board are inserted into the control circuit to control the motor direction and motor speed. Two potentiometers are installed to monitor the movement of the guide plates to avoid collision. Two strain gauges are installed to measure the forces acting on the motors, and the data are read by a data logger. A capacitive six-axis force-torque sensor is used to measure force and torque loads on the passive side under docking conditions.

The complete test apparatus is shown in Fig. 9.

\section{Operation Test Results}

\subsection{Function Verification Test}

(1) A maximum force load of $200 \mathrm{~N}$ that can be withstood by the prototype model is confirmed.

(2) A maximum torque load of $5 \mathrm{~N} \cdot \mathrm{m}$ that can be withstood by the prototype model is confirmed.

(3) A maximum position misalignment of $21 \mathrm{~mm}$ that can be withstood by the prototype model is confirmed.

(4) A maximum rotation misalignment of $5^{\circ}$ that can be withstood by the prototype model is confirmed.

(5) The velocity of the springs during deployment is 


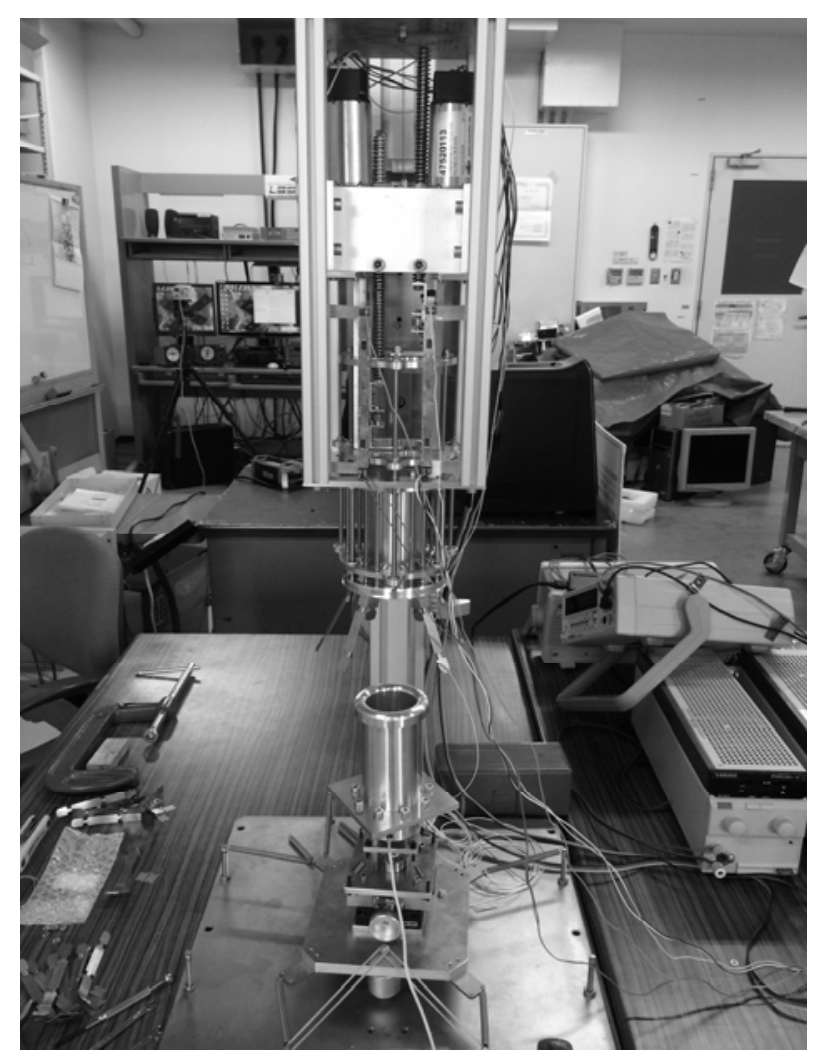

Fig. 9 The complete test apparatus.

$9.3 \mathrm{~mm} / \mathrm{s}$, the velocity of fixing-pins during deployment is $9.0 \mathrm{~mm} / \mathrm{s}$, and the velocity of springs and fixing-pins is $8.9 \mathrm{~mm} / \mathrm{s}$ when they are simultaneously retracted.

4.2 Optimization for the End-Effector Exchange Mechanism

(1) Three types of springs with different thicknesses ( $0.3 \mathrm{~mm}, 0.2 \mathrm{~mm}$, and $0.1 \mathrm{~mm}$ ) are tested.

(2) Three patterns of the number of springs $(9,6$, and 3) are tested.

(3) Two types of passive sides with edges of different thicknesses are tested.

(4) Two different positions of the latching points are tested.

Based on a comparison of the above test results, a six-spring set-up with $0.3 \mathrm{~mm}$ spring thickness, a thin-edged passive side, and a high latching position is selected as the optimal combination.

The optimization of the prototype model is also generalized to other EEEMs of the spring-capture type.
This is summarized as follows:

(1) Type of springs

If there is no plastic deformation of the springs when they are closed by external mechanisms, springs with maximum possible thickness which means springs with the maximum possible coefficient of elasticity are desirable.

(2) Number of springs

The angle between the direction of misalignment and spring should not be too large that leads to an ideal number of springs, either 6 or 7 .

(3) Shape of the passive side

A thin-edged passive side is preferred because it exhibits a better docking performance and weighs less than the thick-edged passive side.

(4) Position of latching points

A higher latching position is preferred because it exhibits a better docking performance. However, it should be calculated such that it satisfies the system requirements for misalignment tolerance.

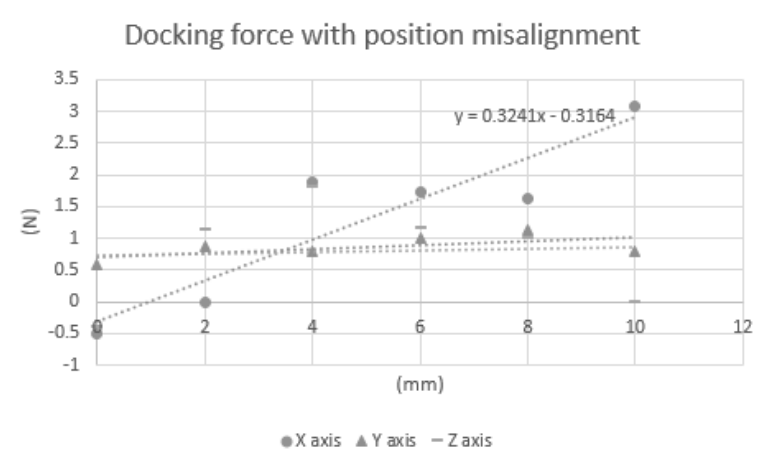

Fig. 10 The relation between docking force and position misalignment.

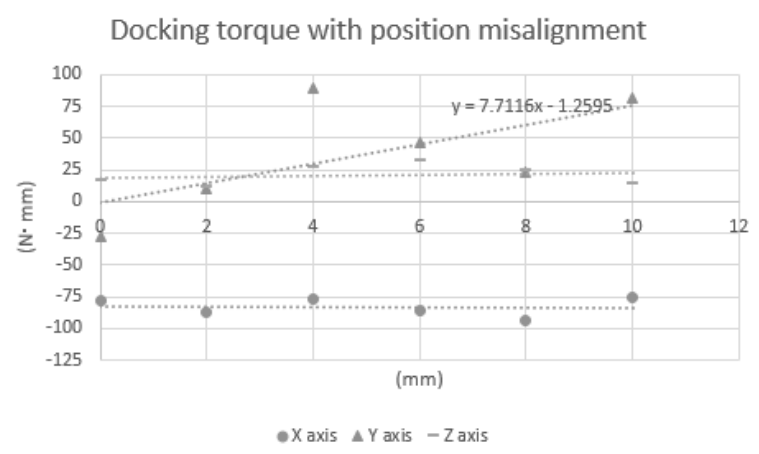

Fig. 11 The relation between docking torque and position misalignment. 


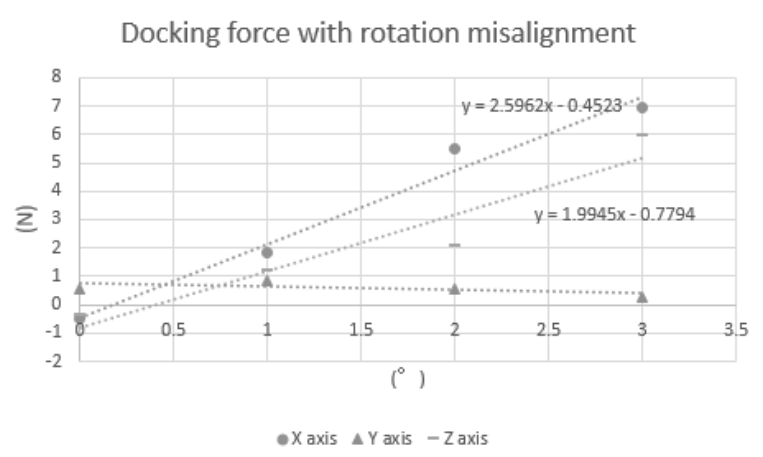

Fig. 12 The relation between docking force and rotation misalignment.

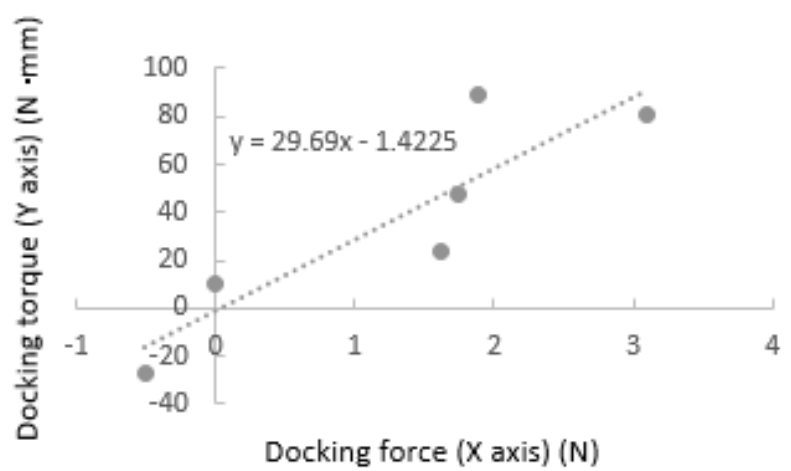

Fig. 14 The relation between docking force and docking torque (with position misalignment).
Docking torque with rotation misalignment

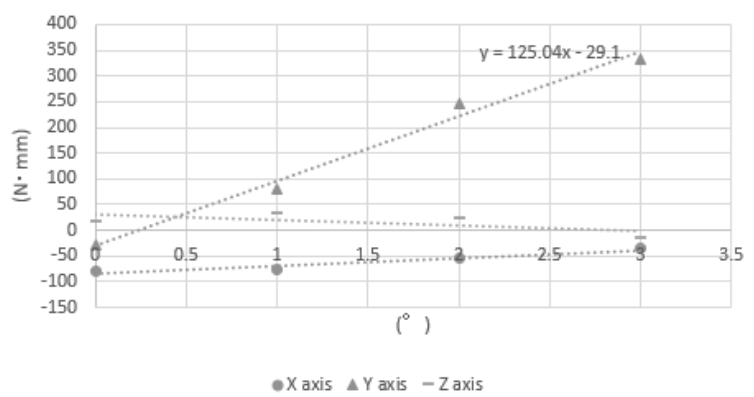

Fig. 13 The relation between docking torque and rotation misalignment.

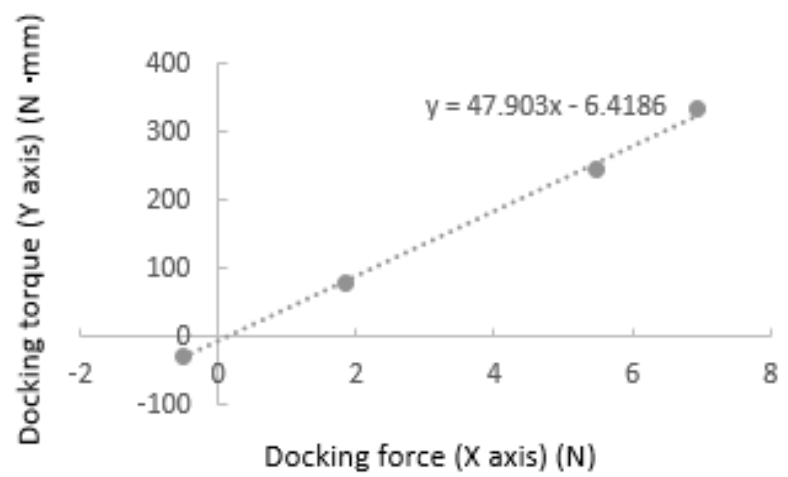

Fig. 15 The relation between docking force and docking torque (with rotation misalignment).

Table 3 Force acting on motors with different rotation misalignment.

\begin{tabular}{llll}
\hline Rotation misalignment $\left(^{\circ}\right)$ & 0 & 1 & 2 \\
\hline Maximum docking force (spring) (N) & 51.87 & 55.92 & 58.79 \\
Maximum docking force (fixing-pin) (N) & 35.45 & 36.76 & 36.81 \\
Holding force (spring) (N) & 21.69 & 26.25 & 30 \\
Holding force (fixing-pin) (N) & 31.22 & 31.89 & 26 \\
\hline
\end{tabular}

(5) Shape of the latching mechanisms

Furthermore, additional improvement for latching mechanisms should be considered to increase the misalignment tolerance.

\subsection{A Study of the Relation between Docking Force and Misalignment}

(1) Docking force and torque act on the passive side, and those obtained with position misalignments of $0 \mathrm{~mm}$, $2 \mathrm{~mm}, 4 \mathrm{~mm}, 6 \mathrm{~mm}, 8 \mathrm{~mm}$, and $10 \mathrm{~mm}$ and rotation misalignments of $0^{\circ}, 1^{\circ}, 2^{\circ}$, and $3^{\circ}$ are compared.

(2) Maximum docking force and holding force act on motors, and those obtained with rotation misalignments of $0^{\circ}, 1^{\circ}$, and $2^{\circ}$ are compared.
The test results are shown in Figs. 10-15 and Table 3 .

Docking force in the direction of the $\mathrm{X}$ axis and docking torque in the direction of the $\mathrm{Y}$ axis increase linearly with position as well as rotation misalignment. A comparison of the relation between docking force in the direction of the $\mathrm{X}$ axis and docking torque in the direction of the $\mathrm{Y}$ axis with two the types of misalignment indicates that the proportional coefficient with the rotation misalignment exceeds that with the position misalignment.

There is no linear relation between the force acting on the motors and rotation misalignment. This is because an increase in the misalignment (in this test, it 
implies that the angle of rotation misalignment gets to $2^{\circ}$ ) leads to a back angle (in this test, it is $0.5^{\circ}$ ) at the rotation stage.

A method of misalignment canceling for the robot arm control is proposed, based on the above test results. For a known misalignment at the passive side (end-effector side), the active side (robot arm side) cancels it as follows:

- For position misalignment

A horizontal locomotion is sufficient to cancel position misalignment.

- For rotation misalignment

In practice, rotation misalignment occurs at the passive side, instead of occurring at the active side as shown in the test. Thus, horizontal locomotion as well as rotation is necessary, and it is necessary to calculate the displacement of locomotion in advance.

- Back angle occurring at the rotation stage

The relation between the back angle and original misalignment should be examined further.

\section{Conclusions}

This study proposes a space construction mission and an EEEM of a robot arm that is necessary for the space construction missions. A new concept of an EEEM that utilizes a spring mechanism to enlarge misalignment tolerance is proposed. A prototype model is developed, and operation tests are performed. A future study will involve the method of robot arm control and a solution for the regolith problem.

\section{References}

[1] Oda, M., Nishida, S., and Nishida. M. 1996. "Development of an EVA and End-Effector, Grapple Fixtures and Tools for the Satellite Mounted Robot System.” In Proceedings of 1996 IEEE/RSJ International Conference on Intelligent Robots and Systems (IROS), 1536-43.

[2] Nishida, S., Inagaki, T. et al. 1999. "ETS-VII Robot Experiment System." Journal the Robotics Society of Japan 17 (8): 1062-6.

[3] Coleshill, E., Oshinowo, L., Rembala, R., Bina, B., Rey, D., and Sindelar. S. 2009. "Dextre: Improving Maintenance Operations on the International Space Station.” Acta Astronautica 64 (9-10): 869-74.

[4] Rubinger, B., Fulford, P., and Gregoris, L. 2001. "Self -Adapting Robotic Auxiliary Hand (SARAH) for SPDM Operations on the International Space Station." In Proceedings of the 6th International Symposium on Artificial Intelligence and Robotics \& Automation in Space (i-SAIRAS). 